\title{
Tumor microenvironment and breast cancer survival: combined effects of breast fat, M2 macrophages and hyaluronan create a dismal prognosis
}

\author{
Satu Tiainen ${ }^{1,2}\left(\mathbb{D} \cdot\right.$ Amro Masarwah $^{3} \cdot$ Sanna Oikari $^{4} \cdot$ Kirsi Rilla $^{4} \cdot$ Kirsi Hämäläinen $^{5,6,7} \cdot$ Mazen Sudah $^{3}$. \\ Anna Sutela ${ }^{3} \cdot$ Ritva Vanninen $^{3,8} \cdot$ Juho Ikonen $^{1,2} \cdot$ Raija Tammi $^{4} \cdot$ Markku Tammi $^{4} \cdot$ Päivi Auvinen $^{1,2}$
}

Received: 31 August 2019 / Accepted: 5 November 2019 / Published online: 12 November 2019

(c) The Author(s) 2019

\begin{abstract}
Purpose Tumor microenvironment, including inflammatory cells, adipocytes and extracellular matrix constituents such as hyaluronan (HA), impacts on cancer progression. Systemic metabolism also influences tumor growth e.g. obesity and type 2 diabetes (T2D) are risk factors for breast cancer. Here, in 262 breast cancer cases, we explored the combined impacts on survival of M2-like tumor associated macrophages (TAMs), the abundance of breast fat visualized as low density in mammograms, and tumor HA, and their associations with T2D.

Methods Mammographic densities were assessed visually from the diagnostic images and dichotomized into very low density (VLD, density $\leq 10 \%$, "fatty breast") and mixed density (MID, density $>10 \%$ ). The amounts of TAMs (CD163+ and CD68+) and tumor HA were determined by immunohistochemistry. The data of T2D was collected from the patient records. Statistical differences between the parameters were calculated with Chi square or Mann-Whitney test and survival analyses with Cox's model.

Results A combination of fatty breasts (VLD), abundance of M2-like TAMs (CD163+) and tumor HA associated with poor survival, as survival was $88-89 \%$ in the absence of these factors but only $40-47 \%$ when all three factors were present $(p<0.001)$. Also, an association between T2D and fatty breasts was found $(p<0.01)$. Furthermore, tumors in fatty breasts contained more frequently high levels of M2-like TAMs than tumors in MID breasts $(p=0.01)$.

Conclusions Our results demonstrate a dramatic effect of the tumor microenvironment on breast cancer progression. We hypothesize that T2D as well as obesity increase the fat content of the breasts, subsequently enhancing local pro-tumoral inflammation.
\end{abstract}

Keywords Breast cancer $\cdot$ Macrophage $\cdot$ Breast density $\cdot$ Hyaluronan $\cdot$ Diabetes $\cdot$ Obesity

Electronic supplementary material The online version of this article (https://doi.org/10.1007/s10549-019-05491-7) contains supplementary material, which is available to authorized users.

Markku Tammi and Päivi Auvinen: shared authorship.

Satu Tiainen

satu.tiainen@kuh.fi

1 Cancer Center, Kuopio University Hospital, P.O. Box 100, 70029 Kuopio, Finland

2 Institute of Clinical Medicine, University of Eastern Finland, P.O. Box 1627, 70211 Kuopio, Finland

3 Imaging Center, Clinical Radiology, Kuopio University Hospital, P.O. Box 100, 70029 Kuopio, Finland

4 Institute of Biomedicine, University of Eastern Finland, P.O.Box 1627, 70211 Kuopio, Finland
5 Imaging Center, Clinical Pathology, Kuopio University Hospital, P.O. Box 100, 70029 Kuopio, Finland

6 Institute of Clinical Medicine, Clinical Pathology and Forensic Medicine, University of Eastern Finland, P.O. Box 1627, 70211 Kuopio, Finland

7 Biocenter Kuopio and Cancer Center of Eastern Finland, University of Eastern Finland, P.O. Box 1627, 70211 Kuopio, Finland

8 Institute of Clinical Medicine, Clinical Radiology, University of Eastern Finland, P.O. Box 1627, 70211 Kuopio, Finland 


\section{Introduction}

Obesity is a major global problem and often a consequence of the Western lifestyle with high-energy diet and low level of physical activity. Obese individuals commonly develop insulin resistance, a condition that precedes type 2 diabetes (T2D). Obesity and T2D are both risk factors for breast cancer and they also predispose breast cancer patients to a poor outcome. [1-6].

In obese individuals, adipocytes in breast tissue induce the recruitment of inflammatory cells, especially macrophages [7], which in turn maintain a low-level inflammation [8]. Crown-like structures (CLSs) i.e. macrophages located around dead adipocytes are regarded as biomarkers of this white adipose tissue inflammation [9]. Among breast cancer patients, a high level of CLSs in breast tissue associates with a poor outcome [10]. Indeed, chronic low-level inflammation is one of the hallmarks of cancer [11], and several studies have shown, that high numbers of tumor associated macrophages (TAMs) correlate with tumor aggressiveness and a poor outcome in breast cancer $[12,13]$. In addition, a positive correlation has been detected between body mass index (BMI) and the amount of TAMs in the breast cancer microenvironment [13].

Hyaluronan (HA) is a large extracellular glycosaminoglycan and a very interesting molecule in terms of obesity, T2D and inflammation since its synthesis depends on the availability of UDP-sugars, which increase in conjunction with high glucose uptake [14], and the expression of hyaluronan synthases HAS1-3 [15] upregulated by the cytokines and growth factors released during inflammation. Consequently, HA is abundant at sites of inflammation, and mainly via its receptors CD44 and RHAMM, HA can modulate inflammatory responses $[16,17]$ including the recruitment of macrophages in both adipose tissue [18] and breast cancer [13, 19]. Moreover, it seems that HA can induce macrophage polarization into the pro-tumoral M2-like phenotype [20, 21]. HA facilitates breast cancer progression [22] and interestingly, the abundance of tumor HA and obesity exhibit a mutual correlation in breast cancer patients [23].

Mammographic breast density (MBD) describes the composition of breast tissue i.e. the relative proportions of fibroglandular and adipose tissues. A high MBD is a wellestablished risk factor for breast cancer [24, 25] but the impact of MBD on breast cancer survival is less clear since the findings in different studies have been conflicting [25]. It was previously suggested that breast cancer patients with very low density breasts (VLD, density $\leq 10 \%$ ), i.e. breasts abundant with fat, had a poor outcome, and VLD represented a significant negative prognostic factor even after correcting for potential confounding factors including age, menopausal status and BMI [26]. Interestingly, VLD correlated also with a high tumor HA content [27].

In the present material of 262 breast cancer patients, we explored the combined impacts on survival of fatty breasts (VLD), M2-like (CD163+) TAMs and tumor HA, and their possible associations with T2D. We hypothesized that fatty breasts, numerous M2-like TAMs and HA abundance together would create inflammatory conditions that promote tumor progression, resulting in poor outcome. In addition, we hypothesized that the disturbed energy metabolism encountered in T2D and obesity would favor this protumoral inflammation in the tumor microenvironment.

\section{Materials and methods}

\section{Patient material}

The primary material of this retrospective study consisted of 278 breast cancer cases, of which 262 patients with data available of both MBD and the amount of TAMs were included in this study. The patients had been operated due to breast cancer at Kuopio University Hospital during the years 2002-2008. Half of the cases were HER2 positive and half were HER2 negative with matching age and time of surgery [23]. The follow-up data were updated in September 21, 2016. The Ethics Committee of the University of Eastern Finland (February 24, 2009, 19//2009), and the National Supervisory Authority for Welfare and Health (VALVIRA, April 8, 2009, 1928/05.01.00.06/2009) provided ethical approval for this study. The study was conducted in accordance with the Declaration of Helsinki.

\section{Type 2 diabetes and obesity}

The data of types 1 and 2 diabetes at the time of breast cancer diagnosis were collected retrospectively from the patient records. Height and weight of the patients were measured for the primary breast cancer operation and collected from anesthesia forms, providing reliable preoperative values [23]. BMI was calculated by the following formula: bodyweight (kg) divided by square of the height $(\mathrm{m})$. According to the WHO classification obesity was determined as BMI $\geq 30 \mathrm{~kg} /$ $\mathrm{m}^{2}$.

\section{Mammographic breast density}

Mammograms were available for 270 patients and MBD was evaluated from the cranio-caudal projections of the original diagnostic mammograms, as reported previously [26]. Briefly, the percentage of the area occupied by radiologically dense breast tissue in the mammogram was evaluated visually. For this study, breast densities of $\leq 10 \%$ were 
classified as VLD ("fatty breast") and $>10 \%$ as mixed densities (MID).

\section{Tumor associated macrophages}

CD163 positivity was regarded as an indicator for M2-like TAMs, and CD68 positivity as an indicator for all TAMs. Immunohistochemical staining for TAMs and their evaluation were performed as described previously [13] with 276 and 270 adequate CD163 and CD68 immunostained tissue sections, respectively, available for analysis. Briefly, three investigators counted the TAMs in at least four hot spots and the average value represented the number of TAMs in the section. The levels of CD163+ and CD68+ TAMs were classified as either low or high; values lower or equal than the median were graded as "low", and values higher than the median as "high".

\section{Hyaluronan}

The stainings of HA in breast carcinoma cells and adjacent stroma were performed as reported earlier [23]. In the statistical analyses, HA in breast carcinoma cells was classified as weak or strong ( $\leq 50 \%$ and $>50 \%$ stained cells, respectively), and stromal HA as weak or strong according to the intensity of the staining.

\section{The standard histopathological factors}

Tumor size, nodal status, histopathological grade and type of the tumor (i.e. ductal, lobular etc.), estrogen (ER) and progesterone (PR) receptor status (immunohistochemistry) and HER2 expression (chromogenic in situ hybridization test) were determined in Kuopio University Hospital, Department of Pathology at the time of diagnosis in accordance with the WHO and international guidelines [28].

\section{Statistical analyses}

The statistical analyses were performed with IBM SPSS Statistics 22 for Windows (IBM Corporation, Armonk, NY, USA). Chi square test and Mann-Whitney test were utilized to calculate the differences between the parameters. Univariate survival analyses were calculated with Cox's model, and survival curves were plotted with the Kaplan-Meier method. Cox's model was used also for multivariate survival analyses; the variables included were MBD, CD163+ and CD68+ TAMs, HA in breast carcinoma cells, stromal HA, BMI, T2D, tumor size (T2-4 vs. T1), nodal status (N1-3 vs. N0), ER and HER2 status. Overall survival (OS) and breast cancer specific survival (BCSS) were calculated from the date of diagnosis to death or end of follow-up; death from any cause was included as an event for OS and death from breast cancer for BCSS. Disease free survival (DFS) was calculated from the date of diagnosis to disease recurrence, death or end of follow-up; only disease recurrence was included as an event. $p$ values $\leq 0.05$ were considered statistically significant.

\section{Results}

\section{Characteristics of the cases}

The clinicopathological parameters of the 262 cases with data available of all the assays are presented in Tables 1 and 2. The median follow-up time was 9.7 years (range $0.5-15.2$ years). During the follow up, 78 patients $(30 \%)$ had a relapse and 63 (24\%) developed distant metastases. Overall, 70 patients (27\%) had died; 52 of them due to breast cancer and 18 from other causes.

Among the 262 cases, 37\% had VLD breasts and the remaining 63\% had MID breasts (Table 2). High levels of

Table 1 The standard histopathological parameters

\begin{tabular}{|c|c|}
\hline \multicolumn{2}{|c|}{ Tumor classification, $n(\%)$} \\
\hline pT1 & $145(55 \%)$ \\
\hline pT2 & $94(36 \%)$ \\
\hline pT3 & $10(4 \%)$ \\
\hline pT4 & $13(5 \%)$ \\
\hline \multicolumn{2}{|c|}{ Nodal classification, $n(\%)$} \\
\hline pNO & $96(37 \%)$ \\
\hline $\mathrm{pN} 1$ & $116(44 \%)$ \\
\hline $\mathrm{pN} 2$ & $34(13 \%)$ \\
\hline pN3 & $16(6 \%)$ \\
\hline \multicolumn{2}{|c|}{ Histological grade, $n(\%)$} \\
\hline 1 & $22(8 \%)$ \\
\hline 2 & $113(43 \%)$ \\
\hline 3 & $127(49 \%)$ \\
\hline \multicolumn{2}{|c|}{ Tumor histology, $n(\%)$} \\
\hline Ductal & $214(82 \%)$ \\
\hline Lobular & $26(10 \%)$ \\
\hline Mucinous & $4(1 \%)$ \\
\hline Other & $18(7 \%)$ \\
\hline \multicolumn{2}{|c|}{ HER2 status, $n(\%)$} \\
\hline Positive & $129(49 \%)$ \\
\hline Negative & $133(51 \%)$ \\
\hline \multicolumn{2}{|c|}{ ER status, $n(\%)$} \\
\hline Positive & $188(72 \%)$ \\
\hline Negative & $74(28 \%)$ \\
\hline \multicolumn{2}{|c|}{ PR status, $n(\%)$} \\
\hline Positive & $163(62 \%)$ \\
\hline Negative & $99(38 \%)$ \\
\hline
\end{tabular}

$E R$ estrogen receptor, $P R$ progesterone receptor 
Table 2 Characteristics of the cases

\begin{tabular}{ll}
\hline Age, years & \\
Median & 58.7 \\
Range & $32-86$ \\
BMI $\left(\mathrm{kg} / \mathrm{m}^{2}\right), n(\%)$ & \\
$<30$ & $207(79 \%)$ \\
$\geq 30$ & $55(21 \%)$ \\
T2D, $n(\%)$ & \\
No & $227(87 \%)$ \\
Yes & $35(13 \%)$ \\
Relapse, $n(\%)$ & \\
No & $184(70 \%)$ \\
Yes & $78(30 \%)$ \\
Death, $n(\%)$ & \\
No & $192(73 \%)$ \\
Yes & $70(27 \%)$ \\
MBD, $n(\%)$ & \\
MID & $166(63 \%)$ \\
VLD & $96(37 \%)$ \\
CD163+ TAMs, $n(\%)$ & \\
Low & $134(51 \%)$ \\
High & $128(49 \%)$ \\
CD68 + TAMs, $n(\%)$ & $126(48 \%)$ \\
Low & \\
High & \\
\hline
\end{tabular}

$B M I$ body mass index, $T 2 D$ type 2 diabetes, $M B D$ mammographic breast density; MID mixed density, $V L D$ very low density, TAMs tumor associated macrophages

M2-like (CD163+) TAMs were detected in 49\% and high levels of CD68+ TAMs in $48 \%$ of the tumors (Table 2). T2D was present in $13 \%$ of the patients; none of the patients had type 1 diabetes (Table 2$)$. Half $(51 \%, n=18)$ of the type 2 diabetics were also obese. Of the T2D patients, 66\% $(n=23)$ were treated with metformin (19 only metformin and 4 metformin with insulin), $6 \%(n=2)$ with other oral T2D medication, $3 \%(n=1)$ with insulin alone, $23 \%(n=8)$ were not receiving medication for T2D and for one patient the information of T2D treatments was missing. At the time of diagnosis, $21 \%$ of all the patients were obese (BMI $\geq 30)$ (Table 2). The standard pathological factors such as tumor size, nodal status, hormone receptor status and HER2 status were similar among the obese and non-obese patients (data not shown). In line with previous data [26], an association was found between obesity and VLD breasts $(p<0.01)$ (Table 3$)$. Also, the median for the number of CD163+ TAMs was higher in tumors of obese compared to tumors of nonobese patients, 29 (range 10-58) and 25 (range 5-65), respectively $(p=0.032)$, but there was no correlation between CD68+ TAMs and BMI $(p=0.6)$.
Table 3 The correlations between breast density and TAMs, T2D and BMI

\begin{tabular}{clcc}
\hline & $\begin{array}{l}\text { VLD } \\
n=96 \\
n(\%)\end{array}$ & $\begin{array}{l}\text { MID } \\
n=166 \\
n(\%)\end{array}$ & $p$ value \\
& & & \\
\hline CD163+ TAMs & $39(41 \%)$ & $95(57 \%)$ & \\
Low $(n=134)$ & $57(59 \%)$ & $71(43 \%)$ & 0.01 \\
High $(n=128)$ & & & \\
CD68+TAMs & $49(51 \%)$ & $79(52 \%)$ & \\
Low $(n=136)$ & $47(49 \%)$ & $79(48 \%)$ & 0.831 \\
High $(n=126)$ & $70(73 \%)$ & $157(95 \%)$ & \\
T2D & $26(27 \%)$ & $9(5 \%)$ & $<0.01$ \\
No $(n=227)$ & & & \\
Yes $(n=35)$ & $63(66 \%)$ & $144(87 \%)$ & \\
BMI $\left(\mathrm{kg} / \mathrm{m}^{2}\right)$ & $33(34 \%)$ & $22(13 \%)$ & $<0.01$ \\
$<30(n=207)$ & & & \\
$\geq 30(n=55)$ & & & \\
\hline
\end{tabular}

TAMs tumor associated macrophages, BMI body mass index, T2D type 2 diabetes, $V L D$ very low density, $M I D$ mixed density

\section{High level of M2-like TAMs associates with VLD breasts}

Tumors in VLD breasts contained more often high levels of M2-like TAMs than tumors in MID breasts, i.e. 59\% and $43 \%$, respectively $(p=0.01)$ (Table 3$)$. Similarly, among the non-obese patients $(n=207)$, high levels of M2-like TAMs occurred in $57 \%$ and $41 \%$ of the tumors in VLD and in MID breasts, respectively $(p=0.032)$. Among the obese patients $(n=55)$, high levels of M2-like TAMs occurred frequently both in the tumors in VLD and in MID breasts, i.e. $64 \%$ and $55 \%$, respectively (ns). No correlation was found between breast density and the amount of CD68+ TAMs (ns) (Table 3).

\section{Type 2 diabetes associates with VLD breasts}

An association was found between T2D and VLD breasts, since $27 \%$ of the patients with VLD breasts but only $5 \%$ of the patients with MID breasts had T2D $(p<0.01)$ (Table 3$)$. Among the non-obese patients, T2D was found in 19\% and $4 \%$ of the patients with VLD and MID breasts, respectively $(p<0.01)$. Among the obese patients, T2D occurred in $42 \%$ and $18 \%$ of the patients with VLD and MID breasts, respectively $(p=0.061)$. No correlations were found between T2D and the amounts of TAMs or HA (data not shown). A non-significant trend towards lower tumor HA among T2D patients with metformin medication $(n=23)$ compared to those without metformin $(n=11)$ was found, but there were no correlations between TAMs and T2D treatments (Supplementary Table S1). 


\section{Fatty breasts (VLD), a high level of M2-like TAMs and high tumor HA create a risk for dismal outcome}

In line with previous data [13, 26], OS and DFS were inferior in patients with VLD breasts as compared to MID breasts ( $p=0.001$ and $p<0.001)$, and with a high level of M2-like TAMs as compared to a low level $(p=0.001)$. Of the 57 patients with VLD breasts and a high level of M2-like TAMs, only $54 \%$ were alive at the end of the follow up as compared to $86 \%$ of the 95 patients with MID breasts and a low level of M2-like TAMs $(p<0.01)$; in other words, patients with VLD breasts and a high level of M2-like TAMs had a 4.4 times higher mortality risk (Fig. 1a, Table 4). Similarly, the risk for breast cancer recurrence was 3.7 times greater among patients with VLD breasts and a high level of M2-like TAMs as compared to patients with neither of these factors, and accordingly DFS rates were lower (Table 4, Fig. 1b). Patients with one of these unfavorable prognostic factors, i.e. VLD breasts or a high level of M2-like TAMs, had mediocre OS and DFS rates (Fig. 1a, b, Table 4).

Even poorer survival was seen among patients with VLD breasts, a high level of M2-like TAMs and high HA expression either in stromal $(n=47)$ or in breast carcinoma cells $(n=38)$, the risk for death being 6.5-7.8 times greater, and OS rates $40-47 \%$ versus $88-89 \%$, when compared to patients with none of these factors $(p<0.001)$ (Fig. 1c, e, Table 4). The DFS rates were also dismal among patients with all three unfavorable factors $(p<0.001)$ (Fig. 1d, f, Table 4). Thus, HA abundance further increased the risk for an unfavorable outcome conferred by VLD breasts and a high level of M2-like TAMs. The differences in OS and DFS rates according to MBD, M2-like TAMs and tumor HA were similar among the HER2-positive $(n=129)$ and HER2negative $(n=133)$ patients (Supplementary Table S2).

\section{Obesity and type 2 diabetes correlate with poor survival}

Both OS and DFS rates were inferior in the obese individuals as compared to the non-obese, $56 \%$ versus $78 \%$ for OS $(p=0.001)$ and $62 \%$ versus $73 \%$ for DFS ( $p=0.044)$ (Fig. 2a, b, Table 4). BCSS was also inferior among the obese patients ( $p=0.028$, HR 1.94, 95\% CI 1.07-3.49). The OS of the T2D patients was inferior as compared to the other subjects, as the OS rates were $57 \%$ versus $76 \%$, respectively $(p=0.027)$ (Fig. 2c, Table 4). However, there were no statistically significant differences in the DFS rates (Fig. 2d, Table 4).

Of all the patients, $7 \%$ were both obese and had T2D, $14 \%$ were only obese, $6 \%$ had only T2D and $73 \%$ were nonobese and did not suffer from T2D. The OS rates were worse among patients who were only obese and among patients who were both obese and had T2D as compared to patients with neither of these conditions ( $p=0.001$ and $p=0.022$, respectively), with a similar trend apparent in the patients with only T2D ( $p=0.056)$ (Fig. 2e, Table 4$)$. The DFS rate was inferior among the obese patients without T2D in comparison to patients with neither of these conditions $(p=0.011)$, but not among patients who had both obesity and T2D or only T2D (Fig. 2f, Table 4). In summary, while low OS correlated with both obesity and T2D, the DFS rate was inferior only among the obese patients without T2D.

\section{Cox multivariate analyses}

In the Cox multivariate analysis, significant prognostic factors for OS were nodal status, VLD, tumor size, HER2 status, BMI and ER status. For BCSS, significant prognostic factors were nodal status, VLD, tumor size, HER2 status and M2-like TAMs (Table 5).

\section{Discussion}

In this study of 262 breast cancer cases, we found a remarkable risk for death with the combination of fatty breasts (VLD), abundance of M2-like TAMs and tumor HA, demonstrating the importance of tumor microenvironment in breast cancer progression. We also showed that tumors in fatty breasts frequently contain high levels of M2-like macrophages, which facilitate pro-tumoral low-level inflammation. Fatty breasts associate with obesity and in the present study also with T2D, suggesting that the metabolic disturbance present in obesity and T2D may promote these local conditions favorable for tumor growth.

Tumors arising in fatty breasts are particularly abundant with HA [27] and here with M2-like TAMs, both indicators of chronic inflammation. M2-like macrophages facilitate low-level inflammation and tissue remodeling required for tumor growth [29]. HA in the peritumoral matrix promotes tumor cell invasion and shields them against immune attack, while HA fragments signal for an exacerbation of inflammation [22]. Moreover, HA synthesized under conditions of cellular stress can coalesce into cable-like structures that recruit macrophages [17] and induce their polarization towards the M2-phenotype [20]. What makes breast fat such a good platform for this cancer-promoting inflammation, remains a question to be answered in later studies.

Fatty breasts associate with obesity, and interestingly in the present study fatty breasts were found more often also in T2D patients even if they were not obese. Obesity associates also with a high level of M2-like TAMs [13] and tumor HA [23], and it is difficult to discern whether one of these factors is the primary effector in breast cancer progression with the other factors following as a consequence. In COX multivariate analyses including all of these factors, VLD 

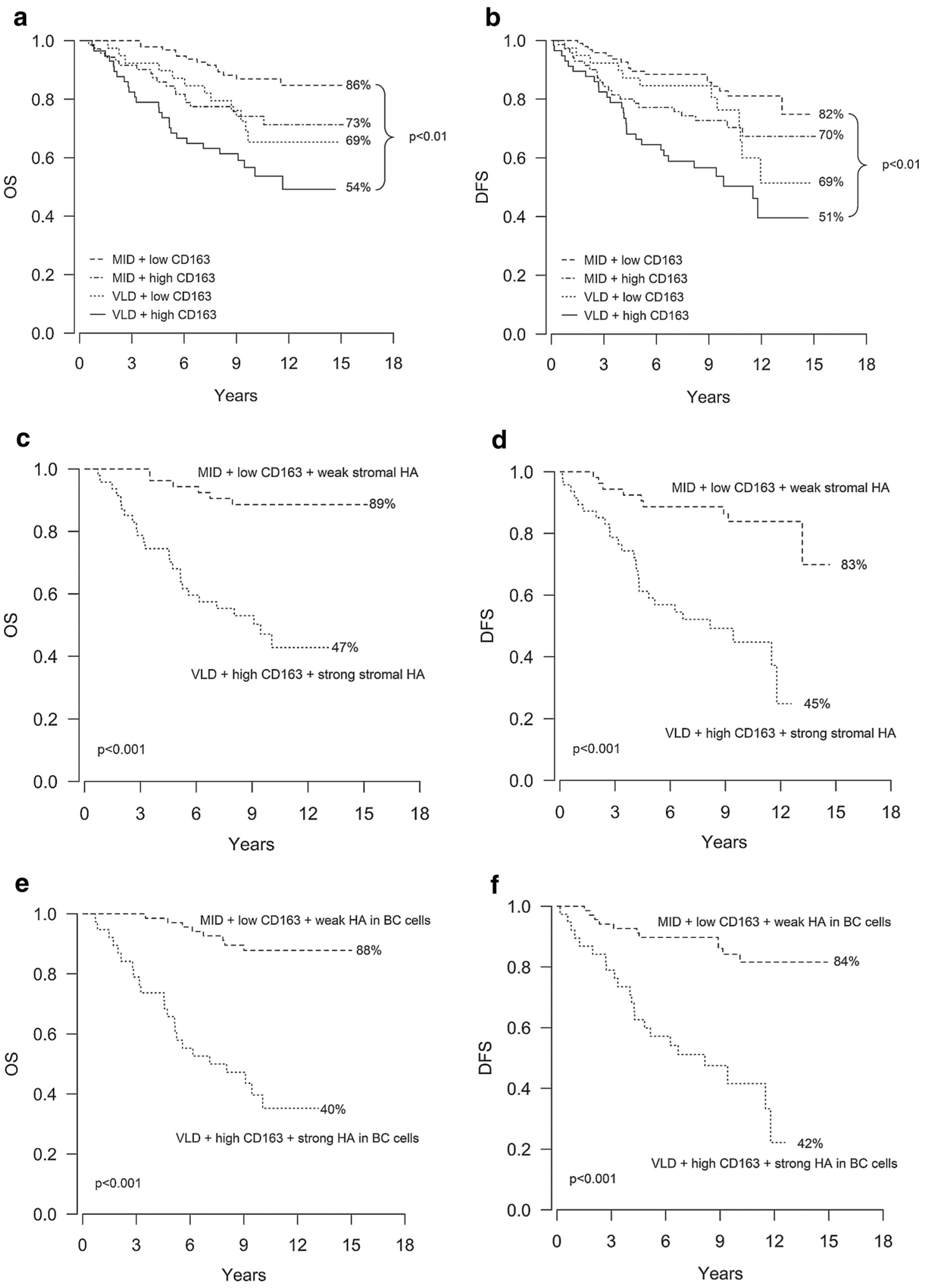

Fig. 1 The combination of VLD breasts, abundance of M2-like TAMs and HA create a dismal survival. Kaplan-Meier curves showing overall survival (OS) and disease free survival (DFS) according to breast density and the level of M2-like (CD163+) TAMs (a, b);

breast density, the level of M2-like (CD163+) TAMs and stromal HA (c, d) and breast density, the level of M2-like (CD163+) TAMs and HA in breast cancer cells (e, f) 
Table 4 Survival analyses

\begin{tabular}{|c|c|c|c|c|c|c|c|c|}
\hline & OS $(\%)$ & $p$ value & HR & $95 \% \mathrm{CI}$ & DFS & $p$ value & HR & $95 \% \mathrm{CI}$ \\
\hline MID + low CD163 & 86 & & & & 82 & & & \\
\hline MID + high CD163 & 73 & 0.026 & 2.23 & $1.10-4.51$ & 70 & 0.053 & 1.88 & $0.99-3.57$ \\
\hline VLD+ low CD163 & 69 & 0.023 & 2.49 & $1.13-5.45$ & 69 & 0.091 & 1.89 & $0.90-3.97$ \\
\hline VLD+ high CD163 & 54 & $<0.01$ & 4.39 & $2.25-8.54$ & 51 & $<0.01$ & 3.71 & $2.03-6.79$ \\
\hline MID + low CD163+ weak stromal HA & 89 & & & & 83 & & & \\
\hline VLD + high CD163+ strong stromal HA & 47 & $<0.001$ & 6.48 & $2.65-15.82$ & 45 & $<0.001$ & 5.52 & $2.47-12.32$ \\
\hline MID+ low CD163+ weak HA in BC cells & 88 & & & & 84 & & & \\
\hline VLD + high CD163+ strong HA in BC cells & 40 & $<0.001$ & 7.79 & $3.47-17.48$ & 42 & $<0.001$ & 5.66 & $2.71-11.82$ \\
\hline $\mathrm{BMI}<30$ & 78 & & & & 73 & & & \\
\hline $\mathrm{BMI} \geq 30$ & 56 & 0.001 & 2.32 & $1.42-3.81$ & 62 & 0.044 & 1.67 & $1.01-2.77$ \\
\hline T2D no & 76 & & & & 71 & & & \\
\hline T2D yes & 57 & 0.027 & 1.90 & $1.07-3.37$ & 66 & 0.480 & 1.25 & $0.67-2.31$ \\
\hline $\mathrm{BMI}<30 \&$ no $\mathrm{T} 2 \mathrm{D}$ & 80 & & & & 74 & & & \\
\hline Only T2D & 59 & 0.056 & 2.19 & $0.98-4.91$ & 59 & 0.2 & 1.68 & $0.76-3.71$ \\
\hline Only $\mathrm{BMI} \geq 30$ & 57 & 0.001 & 2.58 & $1.44-4.62$ & 57 & 0.011 & 2.08 & $1.18-3.67$ \\
\hline $\mathrm{BMI} \geq 30 \& \mathrm{~T} 2 \mathrm{D}$ & 56 & 0.022 & 2.43 & $1.14-5.21$ & 72 & 0.72 & 1.18 & $0.47-2.97$ \\
\hline
\end{tabular}

$O S$ overall survival, DFS disease free survival, $M I D$ mixed density, $V L D$ very low density, $H A$ hyaluronan, $B C$ breast cancer, $B M I$ body mass index, $T 2 D$ type 2 diabetes

displayed the highest significance. The key role of the peritumoral fat is further stressed by the fact that VLD remains as an independent indicator of survival even when adjusted for BMI, age and menopausal status [26]. Thus, obesity and T2D could be effectors that increase the fat content of the breasts and subsequently enhance pro-tumoral inflammation in the tumor microenvironment.

Nevertheless, there are many ways how obesity can promote breast cancer progression. Especially after menopause fat tissue is a major source of estrogen, a hormone known to promote breast cancer growth [30]. Also, higher leptin/ adiponectin ratio may induce breast cancer growth [31], as well as hyperinsulinemia and elevated levels of insulinlike growth factor 1 (IGF-1) [32]. In addition, large tumor size, lymph node metastases, high tumor grade and possibly delayed diagnostics have been proposed to contribute to the unfavorable prognosis of obese patients [33]. However, in the present study, the standard clinicopathological parameters did not correlate with obesity, and obesity remained as an independent factor for poor OS also when adjusted for these known prognostic factors. Importantly, fat tissue content of HA, its CD44 receptor and accumulation of macrophages are characteristic features of the systemic inflammation that associates with obesity [9, 18]. Adipose tissue inflammation and insulin resistance associate with high CD44 expression [34], while weight loss reduces the expression of CD44 and the amount of macrophages in adipose tissue [35]. In addition, HA level in the blood is increased among obese [36] and T2D patients but, interestingly, not in type 1 diabetes [37]. This suggests that hyperglycemia alone does not account for the inflammation, thus leaving insulin resistance and hyperinsulinemia as possible culprits.

The metabolic dysfunctions such as insulin resistance and hyperinsulinemia that can subsequently develop into T2D are common in obesity. Indeed, in our study every third $(33 \%, 18 / 55)$ obese patient had also T2D. Interestingly, the DFS rate was reduced among the obese patients, but not among the patients with both obesity and T2D. One explanation could be the administration of metformin, as T2D patients treated with metformin have a reduced incidence of several cancers, including breast cancer [38], and there is also evidence that metformin decreases breast cancer mortality [39, 40]. The higher DFS rate of obese patients with T2D compared to those only obese, suggest that metformin medication interferes with some key processes that promote breast cancer progression in obesity. Indeed, metformin not only decreases the levels of glucose and insulin in the circulation, but also reduces the uptake of glucose into cancer cells [41]. One characteristic of cancer cells is their very high glucose uptake and aerobic glycolysis (Warburg effect) [42]. The accumulation of glycolysis intermediates increases glucose flux into the hexosamine biosynthesis pathway resulting in increased level of its end product uridine diphosphate $N$-acetylglucosamine (UDP-GlcNAc) [14]. UDP-GlcNAc is a key substrate in HA synthesis and is involved in $O$-GlcNAcylation, the latter being a protein modification that contributes to cancer cell survival and associates with poor outcome in breast cancer [43]. There is evidence that metformin inhibits glucose consumption of breast cancer cells via reduced hexokinase activity [44] and decreases 

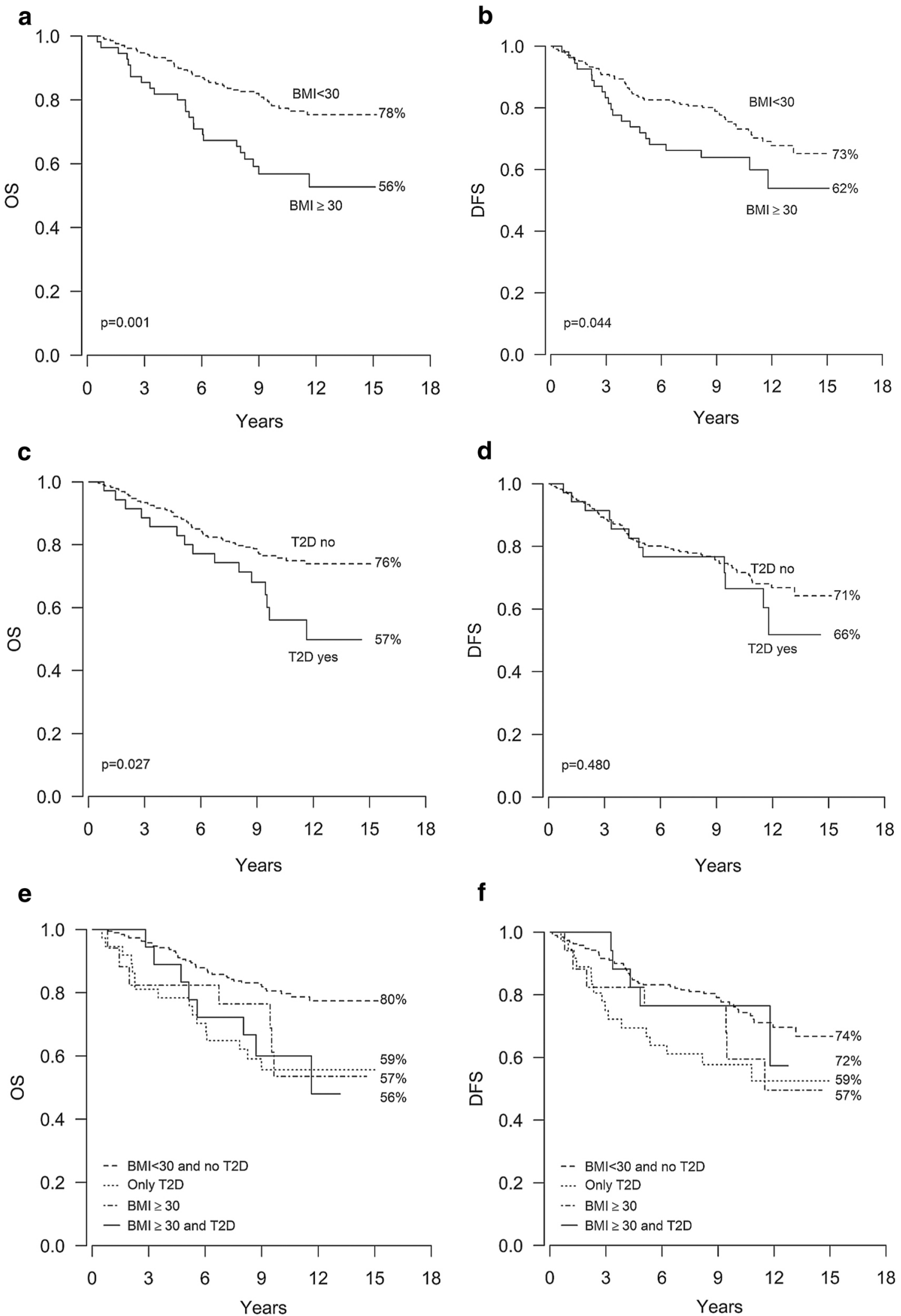

f

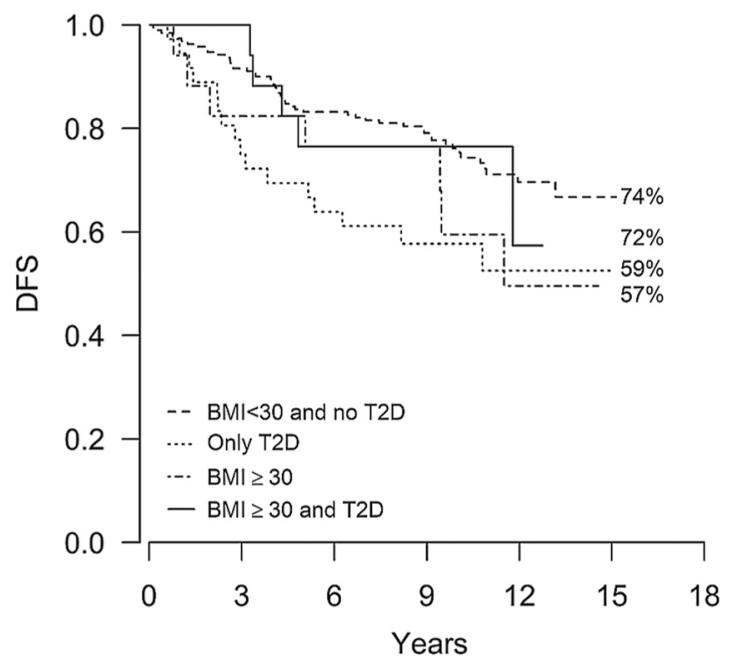

Fig. 2 Obesity and type 2 diabetes correlate with poor overall survival. Kaplan-Meier curves showing overall survival (OS) and disease free survival (DFS) according to BMI (a, b), the presence/absence of T2D $(\mathbf{c}, \mathbf{d})$ and the presence/absence of obesity and T2D (e, f) 
Table 5 COX multivariate analyses for OS and BCSS

\begin{tabular}{|c|c|c|c|}
\hline & $p$ value & HR & $95 \% \mathrm{CI}$ \\
\hline \multicolumn{4}{|l|}{ OS } \\
\hline Nodal status & $<0.001$ & 3.78 & $1.99-7.21$ \\
\hline VLD & $<0.001$ & 2.93 & $1.72-4.97$ \\
\hline Tumor size & $<0.001$ & 2.6 & $1.53-4.42$ \\
\hline HER2 & 0.022 & 1.83 & $1.09-3.08$ \\
\hline BMI & 0.032 & 1.86 & $1.06-3.26$ \\
\hline ER & 0.048 & 0.6 & $0.36-0.996$ \\
\hline CD163+ TAMs & 0.12 & 1.52 & $0.9-2.57$ \\
\hline $\mathrm{HA}$ in $\mathrm{BC}$ cells & 0.27 & 1.38 & $0.78-2.47$ \\
\hline CD68 + TAMs & 0.4 & 0.81 & $0.49-1.33$ \\
\hline Stromal HA & 0.43 & 1.34 & $0.65-2.77$ \\
\hline $\mathrm{T} 2 \mathrm{D}$ & 0.71 & 1.13 & $0.59-2.18$ \\
\hline \multicolumn{4}{|l|}{ BCSS } \\
\hline Nodal status & $<0.001$ & 7.8 & $2.97-20.43$ \\
\hline VLD & $<0.001$ & 3.25 & $1.77-5.95$ \\
\hline Tumor size & 0.001 & 2.99 & $1.54-5.81$ \\
\hline HER2 & 0.022 & 2.06 & $1.11-3.83$ \\
\hline CD163+ TAMs & 0.03 & 2.02 & $1.07-3.83$ \\
\hline ER & 0.06 & 0.57 & $0.32-1.03$ \\
\hline BMI & 0.1 & 1.75 & $0.89-3.45$ \\
\hline Stromal HA & 0.22 & 1.81 & $0.71-4.64$ \\
\hline $\mathrm{HA}$ in $\mathrm{BC}$ cells & 0.42 & 1.32 & $0.68-2.55$ \\
\hline $\mathrm{T} 2 \mathrm{D}$ & 0.66 & 0.83 & $0.36-1.93$ \\
\hline CD68+ TAMs & 0.67 & 0.88 & $0.49-1.58$ \\
\hline
\end{tabular}

$O S$ overall survival, $B C S S$ breast cancer specific survival, $V L D$ very low density, $B M I$ body mass index, $E R$ estrogen receptor, TAMs tumor associated macrophages, $H A$ hyaluronan, $B C$ breast cancer, $T 2 D$ type 2 diabetes

HA synthesis [45]. In addition, metformin can inhibit macrophage polarization into the M2-like phenotype in tumor microenvironment [46] and promote polarization into the M1-phenotype, resulting in the inhibition of tumor growth [47]. Recently it was shown in a rodent model, that metformin inhibited the progression of postmenopausal breast cancer and decreased the amount of macrophages in the tumor microenvironment [48]. Thus, the use of metformin may also explain why there were no correlations found between T2D and the amount of TAMs or HA in the present study. There was a non-significant trend towards lower tumor HA among T2D patients receiving metformin but the number of patients with metformin medication $(n=23)$ in the present study is too small to address this question properly. Prospective trials are ongoing in order to evaluate the effect of metformin on breast cancer outcome.

To conclude, in this study we showed that breast cancers in fatty breasts (VLD) often contain high levels of M2-like TAMs, suggesting that the readily available mammograms may provide important information of tumor biology and microenvironment. The dismal outcome among breast cancer patients with fatty breasts, a high level of M2-like TAMs and high tumor HA emphasize the importance of the local inflammatory conditions for tumor progression. Furthermore, the disturbed energy metabolism encountered in obesity and T2D may increase the fat-content of the breasts and subsequently promote local pro-tumoral inflammation, revealing a potential mechanism that predisposes these patients to a bleak prognosis.

Acknowledgements Open access funding provided by University of Eastern Finland (UEF) including Kuopio University Hospital. We thank Tuomas Selander (Science Service Center, Kuopio University Hospital, Finland) for the help with the statistics; Kari Kotikumpu and Eija Rahunen (Institute of Biomedicine, University of Eastern Finland, Finland) for assistance with the immunohistochemistry, and Ewen MacDonald for English language editing.

Author contributions Material preparation, data collection and analysis were performed by Satu Tiainen, Amro Masarwah and Juho Ikonen. The first draft of the manuscript was written by Satu Tiainen and all authors commented on previous versions of the manuscript with Päivi Auvinen and Markku Tammi as the major contributors in writing the manuscript. All authors contributed to the study conception and read and approved the final manuscript.

Funding This study was supported by the Finnish Society for Oncology and the Special Government Funding (EVO/VTR) of Kuopio University Hospital under Grant No. 5654132. The funders did not participate in the design, conduction or data interpretation of this study.

Data availability The datasets generated and/or analyzed during the current study are not publicly available due to the fact that they contain information that could compromise research participant privacy but may be available from the corresponding author on reasonable request and with required permissions.

\section{Compliance with ethical standards}

Conflict of interest The authors declare that they have no conflict of interest.

Ethical approval All procedures performed in this study were in accordance with the ethical standards of The Ethics Committee of the University of Eastern Finland (February 24, 2009, 19//2009), the National Supervisory Authority for Welfare and Health (VALVIRA, April 8, 2009, 1928/05.01.00.06/2009), and the 1964 Declaration of Helsinki and its later amendments or comparable ethical standards.

Informed consent Informed consent was not required since this is a retrospective study conducted on already available biological material, and obtaining informed consent from all the patients would have been impossible. Ethical approval was provided by the National Supervisory Authority for Welfare and Health (VALVIRA, April 8, 2009, 1928/05.01.00.06/2009).

Open Access This article is distributed under the terms of the Creative Commons Attribution 4.0 International License (http://creativeco mmons.org/licenses/by/4.0/), which permits unrestricted use, distribution, and reproduction in any medium, provided you give appropriate credit to the original author(s) and the source, provide a link to the Creative Commons license, and indicate if changes were made. 


\section{References}

1. Lauby-Secretan B, Scoccianti C, Loomis D, Grosse Y, Bianchini F, Straif K et al (2016) Body fatness and cancer-viewpoint of the IARC working group. N Engl J Med 375:794-798

2. Chan DS, Vieira AR, Aune D, Bandera EV, Greenwood DC, McTiernan A et al (2014) Body mass index and survival in women with breast cancer-systematic literature review and meta-analysis of 82 follow-up studies. Ann Oncol 25:1901-1914

3. Tsilidis KK, Kasimis JC, Lopez DS, Ntzani EE, Ioannidis JP (2015) Type 2 diabetes and cancer: umbrella review of metaanalyses of observational studies. BMJ 350:g7607

4. Zhao XB, Ren GS (2016) Diabetes mellitus and prognosis in women with breast cancer: a systematic review and meta-analysis. Medicine (Baltimore) 95:e5602

5. Barone BB, Yeh HC, Snyder CF, Peairs KS, Stein KB, Derr RL et al (2008) Long-term all-cause mortality in cancer patients with preexisting diabetes mellitus: a systematic review and meta-analysis. JAMA 300:2754-2764

6. Widschwendter P, Friedl TW, Schwentner L, DeGregorio N, Jaeger B, Schramm A et al (2015) The influence of obesity on survival in early, high-risk breast cancer: results from the randomized SUCCESS A trial. Breast Cancer Res 17:129

7. Arendt LM, McCready J, Keller PJ, Baker DD, Naber SP, Seewaldt V et al (2013) Obesity promotes breast cancer by CCL2mediated macrophage recruitment and angiogenesis. Cancer Res 73:6080-6093

8. Russo L, Lumeng CN (2018) Properties and functions of adipose tissue macrophages in obesity. Immunology 155:407-417

9. Cinti S, Mitchell G, Barbatelli G, Murano I, Ceresi E, Faloia E et al (2005) Adipocyte death defines macrophage localization and function in adipose tissue of obese mice and humans. J Lipid Res 46:2347-2355

10. Iyengar NM, Zhou XK, Gucalp A, Morris PG, Howe LR, Giri DD et al (2016) Systemic correlates of white adipose tissue inflammation in early-stage breast cancer. Clin Cancer Res 22:2283-2289

11. Hanahan D, Weinberg RA (2011) Hallmarks of cancer: the next generation. Cell 144:646-674

12. Zhao X, Qu J, Sun Y, Wang J, Liu X, Wang F et al (2017) Prognostic significance of tumor-associated macrophages in breast cancer: a meta-analysis of the literature. Oncotarget 8:30576-30586

13. Tiainen S, Tumelius R, Rilla K, Hamalainen K, Tammi M, Tammi $R$ et al (2015) High numbers of macrophages, especially M2-like (CD163-positive), correlate with hyaluronan accumulation and poor outcome in breast cancer. Histopathology 66:873-883

14. Oikari S, Kettunen T, Tiainen S, Hayrinen J, Masarwah A, Sudah $M$ et al (2018) UDP-sugar accumulation drives hyaluronan synthesis in breast cancer. Matrix Biol 67:63-74

15. Weigel PH, DeAngelis PL (2007) Hyaluronan synthases: a decadeplus of novel glycosyltransferases. J Biol Chem 282:36777-36781

16. Misra S, Hascall VC, Markwald RR, Ghatak S (2015) Interactions between hyaluronan and its receptors (CD44, RHAMM) regulate the activities of inflammation and cancer. Front Immunol 6:201

17. Jokela TA, Kuokkanen J, Karna R, Pasonen-Seppanen S, Rilla $\mathrm{K}$, Kossi J et al (2013) Mannose reduces hyaluronan and leukocytes in wound granulation tissue and inhibits migration and hyaluronan-dependent monocyte binding. Wound Repair Regen 21:247-255

18. Han CY, Subramanian S, Chan CK, Omer M, Chiba T, Wight TN et al (2007) Adipocyte-derived serum amyloid A3 and hyaluronan play a role in monocyte recruitment and adhesion. Diabetes 56:2260-2273

19. Kobayashi N, Miyoshi S, Mikami T, Koyama H, Kitazawa M, Takeoka M et al (2010) Hyaluronan deficiency in tumor stroma impairs macrophage trafficking and tumor neovascularization. Cancer Res 70:7073-7083

20. Kuang DM, Wu Y, Chen N, Cheng J, Zhuang SM, Zheng L (2007) Tumor-derived hyaluronan induces formation of immunosuppressive macrophages through transient early activation of monocytes. Blood 110:587-595

21. Zhang G, Guo L, Yang C, Liu Y, He Y, Du Y et al (2016) A novel role of breast cancer-derived hyaluronan on inducement of M2-like tumor-associated macrophages formation. Oncoimmunology 5:e1172154

22. Tammi MI, Oikari S, Pasonen-Seppanen S, Rilla K, Auvinen $\mathrm{P}$, Tammi RH (2019) Activated hyaluronan metabolism in the tumor matrix: causes and consequences. Matrix Biol 78-79:147-164

23. Auvinen P, Tammi R, Kosma VM, Sironen R, Soini Y, Mannermaa A et al (2013) Increased hyaluronan content and stromal cell CD44 associate with HER2 positivity and poor prognosis in human breast cancer. Int J Cancer 132:531-539

24. Boyd NF, Guo H, Martin LJ, Sun L, Stone J, Fishell E et al (2007) Mammographic density and the risk and detection of breast cancer. N Engl J Med 356:227-236

25. Shawky MS, Huo CW, Henderson MA, Redfern A, Britt K, Thompson EW (2019) A review of the influence of mammographic density on breast cancer clinical and pathological phenotype. Breast Cancer Res Treat 177:251-276

26. Masarwah A, Auvinen P, Sudah M, Rautiainen S, Sutela A, Pelkonen O et al (2015) Very low mammographic breast density predicts poorer outcome in patients with invasive breast cancer. Eur Radiol 25:1875-1882

27. Masarwah A, Tammi M, Sudah M, Sutela A, Oikari S, Kosma VM et al (2015) The reciprocal association between mammographic breast density, hyaluronan synthesis and patient outcome. Breast Cancer Res Treat 153:625-634

28. Leslie H. Sobin, CW (2002) TNM classification of malignant tumours, 6th edn (UICC)

29. Mills CD, Ley K (2014) M1 and M2 macrophages: the chicken and the egg of immunity. J Innate Immun 6:716-726

30. Kaaks R, Rinaldi S, Key TJ, Berrino F, Peeters PH, Biessy C et al (2005) Postmenopausal serum androgens, oestrogens and breast cancer risk: the European prospective investigation into cancer and nutrition. Endocr Relat Cancer 12:1071-1082

31. Grossmann ME, Ray A, Nkhata KJ, Malakhov DA, Rogozina OP, Dogan S et al (2010) Obesity and breast cancer: status of leptin and adiponectin in pathological processes. Cancer Metastasis Rev 29:641-653

32. Bowers LW, Rossi EL, O'Flanagan CH, deGraffenried LA, Hursting SD (2015) The role of the insulin/IGF system in cancer: lessons learned from clinical trials and the energy balancecancer link. Front Endocrinol (Lausanne) 6:77

33. Jiralerspong S, Goodwin PJ (2016) Obesity and breast cancer prognosis: evidence, challenges, and opportunities. J Clin Oncol 34:4203-4216

34. Liu LF, Kodama K, Wei K, Tolentino LL, Choi O, Engleman EG et al (2015) The receptor CD44 is associated with systemic insulin resistance and proinflammatory macrophages in human adipose tissue. Diabetologia 58:1579-1586

35. Bertola A, Deveaux V, Bonnafous S, Rousseau D, Anty R, Wakkach A et al (2009) Elevated expression of osteopontin may be related to adipose tissue macrophage accumulation and liver steatosis in morbid obesity. Diabetes 58:125-133

36. Prakash J, Gabdulina G, Trofimov S, Livshits G (2017) Quantitative genetics of circulating hyaluronic acid (HA) and its correlation with hand osteoarthritis and obesity-related phenotypes in a community-based sample. Ann Hum Biol 44:522-530

37. Nagy N, Sunkari VG, Kaber G, Hasbun S, Lam DN, Speake $\mathrm{C}$ et al (2018) Hyaluronan levels are increased systemically in 
human type 2 but not type 1 diabetes independently of glycemic control. Matrix Biol. https://doi.org/10.1016/j.matbi o.2018.09.003

38. Col NF, Ochs L, Springmann V, Aragaki AK, Chlebowski RT (2012) Metformin and breast cancer risk: a meta-analysis and critical literature review. Breast Cancer Res Treat 135:639-646

39. Zhang P, Li H, Tan X, Chen L, Wang S (2013) Association of metformin use with cancer incidence and mortality: a meta-analysis. Cancer Epidemiol 37:207-218

40. Tang GH, Satkunam M, Pond GR, Steinberg GR, Blandino G, Schunemann HJ et al (2018) Association of metformin with breast cancer incidence and mortality in patients with type II diabetes: a GRADE-assessed systematic review and meta-analysis. Cancer Epidemiol Biomarkers Prev 27:627-635

41. Shlomai G, Neel B, LeRoith D, Gallagher EJ (2016) Type 2 diabetes mellitus and cancer: the role of pharmacotherapy. J Clin Oncol 34:4261-4269

42. Vander Heiden MG, Cantley LC, Thompson CB (2009) Understanding the Warburg effect: the metabolic requirements of cell proliferation. Science 324:1029-1033

43. Tiainen S, Oikari S, Tammi M, Rilla K, Hämäläinen K, Tammi R et al (2016) High extent of $O$-GlcNAcylation in breast cancer cells correlates with the levels of HAS enzymes, accumulation of hyaluronan, and poor outcome. Breast Cancer Res Treat 160:237-247
44. Marini C, Salani B, Massollo M, Amaro A, Esposito AI, Orengo AM et al (2013) Direct inhibition of hexokinase activity by metformin at least partially impairs glucose metabolism and tumor growth in experimental breast cancer. Cell Cycle 12:3490-3499

45. Vigetti D, Clerici M, Deleonibus S, Karousou E, Viola M, Moretto $P$ et al (2011) Hyaluronan synthesis is inhibited by adenosine monophosphate-activated protein kinase through the regulation of HAS2 activity in human aortic smooth muscle cells. J Biol Chem 286:7917-7924

46. Ding L, Liang G, Yao Z, Zhang J, Liu R, Chen H et al (2015) Metformin prevents cancer metastasis by inhibiting M2-like polarization of tumor associated macrophages. Oncotarget 6:36441-36455

47. Wang JC, Sun X, Ma Q, Fu GF, Cong LL, Zhang H et al (2018) Metformin's antitumour and anti-angiogenic activities are mediated by skewing macrophage polarization. J Cell Mol Med. https ://doi.org/10.1111/jcmm.13655

48. Giles ED, Jindal S, Wellberg EA, Schedin T, Anderson SM, Thor AD et al (2018) Metformin inhibits stromal aromatase expression and tumor progression in a rodent model of postmenopausal breast cancer. Breast Cancer Res 20:50

Publisher's Note Springer Nature remains neutral with regard to jurisdictional claims in published maps and institutional affiliations. 